\title{
Screw Theory Based Singularity Analysis of Lower-Mobility Parallel Robots considering the Motion/Force Transmissibility and Constrainability
}

\author{
Xiang Chen, ${ }^{1,2}$ Xin-Jun Liu, ${ }^{1,2}$ and Fugui Xie ${ }^{1,2}$ \\ ${ }^{1}$ State Key Laboratory of Tribology and Institute of Manufacturing Engineering, Department of Mechanical Engineering, \\ Tsinghua University, Beijing 100084, China \\ ${ }^{2}$ Beijing Key Lab of Precision/Ultra Precision Manufacturing Equipments and Control, Tsinghua University, \\ Beijing 100084, China
}

Correspondence should be addressed to Xin-Jun Liu; xinjunliu@mail.tsinghua.edu.cn and Fugui Xie; xiefg@mail.tsinghua.edu.cn Received 14 June 2014; Accepted 10 September 2014

Academic Editor: Carlo Cosentino

Copyright (C) 2015 Xiang Chen et al. This is an open access article distributed under the Creative Commons Attribution License, which permits unrestricted use, distribution, and reproduction in any medium, provided the original work is properly cited.

\begin{abstract}
Singularity is an inherent characteristic of parallel robots and is also a typical mathematical problem in engineering application. In general, to identify singularity configuration, the singular solution in mathematics should be derived. This work introduces an alternative approach to the singularity identification of lower-mobility parallel robots considering the motion/force transmissibility and constrainability. The theory of screws is used as the mathematic tool to define the transmission and constraint indices of parallel robots. The singularity is hereby classified into four types concerning both input and output members of a parallel robot, that is, input transmission singularity, output transmission singularity, input constraint singularity, and output constraint singularity. Furthermore, we take several typical parallel robots as examples to illustrate the process of singularity analysis. Particularly, the input and output constraint singularities which are firstly proposed in this work are depicted in detail. The results demonstrate that the method can not only identify all possible singular configurations, but also explain their physical meanings. Therefore, the proposed approach is proved to be comprehensible and effective in solving singularity problems in parallel mechanisms.
\end{abstract}

\section{Introduction}

In theory, parallel robots, compared with their counterparts, have the potential to answer the increasing need for high stiffness, compactness, load-to-weight ratio, and so forth. With this in mind, extensive attention has been focused on parallel robots. Particularly, most of the parallel robots used successfully in engineering are those with lower mobility. Such engineering applications are nowadays with a rapid rate utilized in precise manufacturing, medical science, space equipment, and others [1].

Before the usage of parallel robots in engineering, singularity, as one significant issue, must be investigated. Singularity is one of the inherent characteristics of parallel robots, which influences a considerable number of performances, including the workspace, dexterity, stiffness, and load capacity. Normally, at singular configurations, a parallel robot loses control over degrees of freedom (DOFs). Either of two effects generally occurs: (1) the robot gains one or more unexpected DOFs, thereby degrading natural stiffness and diminishing load capacity in the direction of the additional DOF or (2) the robot loses one or more DOFs and lies at a dead point where it is uncontrollable. Therefore, the determination of singular configurations, which is critical to understanding a robot's kinematics, should be identified and avoided if possible.

Basically, the singularity is also a typical mathematical problem. The singularity that happened in parallel robots is corresponding to the singular solution in mathematics. In the past three decades, a number of mathematical approaches have been devoted to analyzing the singularity problems in parallel robots. The methods could be typically divided into two categories: analytical and geometric methods. In the analytical methods, the Jacobian matrix of a parallel robot was always derived to identify singularity; that is, 
Gosselin and Angeles [2] identified singularity in closed-loop chains mechanism by analyzing the determinant of Jacobian matrix. The rank deficiency of Jacobian matrix leads to the singularity of a parallel robot [3]. In the geometric methods, screw theory and line geometry were commonly used to solve singularity problems [4]. Park and Kim [5] proposed a coordinate-invariant differential geometric approach to analyzing the singularity in a parallel mechanism. Hunt [6] laid down a general framework for using screw theory in analyzing singularity in parallel robots. Hao and McCarthy [7] concluded the conditions for line-based singularities via the theory of screws. Merlet [8] noticed that it is the possibility to identify all singular configurations in a parallel robot via line geometric method. Ben-Horin Shoham [9] analyzed the singularity of a class of 6-DOF parallel robots using the superbracket and the Grassmann-Cayley operators. More recent approaches to singularity analysis were discussed by Davidson and Hunt [10], Ma and Angeles [11], Bonev et al. [12], and Tsai [13].

The other significant issue in singularity analysis is the classification of singularities. On the basis of singular properties of Jacobian matrices, Gosselin and Angeles [2] classified the singularity into three main groups. With the same definitions, Tsai [13] termed these three kinds of singularities as the inverse, forward, and combined singularities. Gogu [14] distinguished two types of singular configurations: structural and kinematic singularities. Park and Kim [5] classified closed-chain singularities into three basic types: configuration space singularity, actuator singularity, and endeffector singularity. In addition, Liu et al. [15] combined the three groups of singularities proposed by Park to come up with the first-order singularity and established two-order singularities as degenerate and nondegenerate types. Fang and Tsai [16] divided the singularity cases as limb singularity, manipulator singularity, and actuation singularity. Furthermore, Zlatanov et al. [17] considered both the input and output of a parallel robot and concluded that the singularity should always happen for the combination cases among six classified types: redundant input, impossible input, redundant output, impossible output, redundant passive motion, and increased instantaneous mobility. Note that the singularity is an inherent characteristic of parallel robots and not all singularities could be avoided in practice. The elimination of different singularities is another important issue in practice which attracted many researchers. For example, Li and $\mathrm{Xu}$ [18] discussed this problem from the aspect of optimal mechanism design.

However, the predecessors' contributions mainly focused on finding new mathematical tools to search and divide the solutions of singularities. This work, on the other hand, discusses the singularity problem from the view of its practical physical meanings. We put forward an alternative viewpoint to the singularity in terms of the motion/force transmission and constraint properties of lower-mobility parallel robots. As is well known, the key function of a parallel robot is to transmit and constrain the underlying motion and forces between inputs and output. Thereby, in the functional process of a parallel robot, any motion and force, which cannot be transmitted or constrained as expected, could lead to the singularities. Thus, by considering the transmission and constraint properties, we can evaluate the singularities of lower-mobility parallel robots. It should be noted that this work is an intuitive following study with the knowledge of the motion/force transmission and constraint analysis of parallel robots proposed by our research team $[19,20]$. The motion/force transmission concept and its indices were proposed by Wang et al. [19] in 2010, and then the approach to motion/force constraint performance analysis was introduced in 2014 [20]. In both literatures, the indices used to evaluate the transmission and constraint performance of parallel robots have the potential to indicate the worst transmissibility and constrainability when they are equal to zero. In such cases, the mechanism cannot transmit or constrain the underlying motions and forces, thereby resulting in singularities, which are discussed in this work.

The remainder of this paper is organized as follows. Based on the theory of screws, Section 2 proposes a singularity identification approach in terms of the transmission and constraint analysis of lower-mobility parallel robots. Several modified indices are introduced to identify the singularities, and the classification of singularity is discussed based on the indices. In Section 3, several typical parallel robots are taken as examples to demonstrate the usage of the proposed approach and indices. Finally, Section 4 follows with a conclusion of this work.

\section{Approach to Analyzing Singularity and the Classification}

There is no doubt that screw theory is an efficient and profound mathematical tool for studying parallel mechanisms, and it has been used in evaluating the kinematic performance, type synthesis, and singularity analysis [21-25]. We introduce our analytical approach to elucidating singularity and its classifications, in which we consider motion/force transmissibility and constrainability in parallel robots on the basis of screw theory. It should be noted that this work only considers the nonredundant parallel robots with fewer than six DOFs, wherein there exist the constraint motions and forces.

2.1. Mathematical Foundation. In general, a screw can be represented in dual-vector form as follows [26]:

$$
\$=s+\in s_{0}
$$

where real part $s$ is a unit vector in the direction of the screw axis, dual part $s_{0}$ denotes the moment of the screw with respect to the origin, and $\epsilon$ refers to dual unity, which is defined such that

$$
\epsilon \neq 0, \quad \epsilon^{2}=0
$$

Considering one arbitrary point $P$, of position vector $\mathbf{p}$, on the screw axis, we have

$$
\mathbf{s}_{0}=\mathbf{p} \times \mathbf{s}+h \mathbf{s},
$$




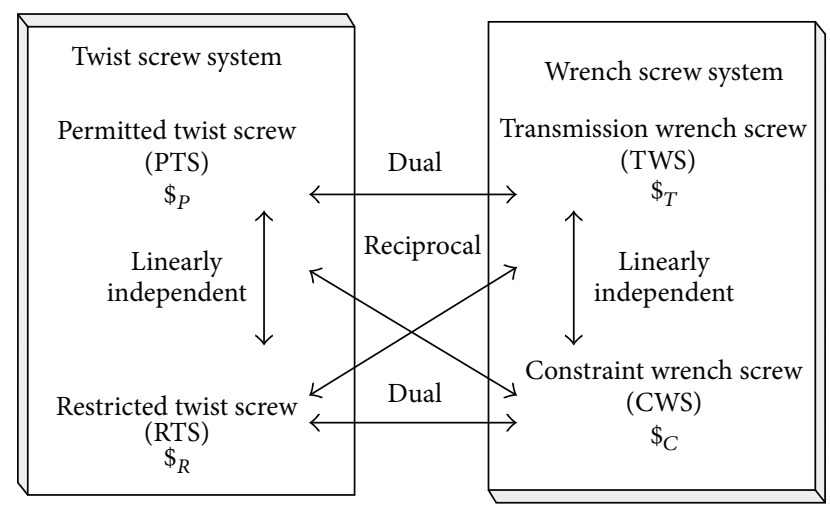

FIGURE 1: Interrelationship among four twist and wrench screw systems.

where $h$ is defined as the pitch of the screw. Here, we get the following relationship:

$$
h=\mathbf{s}^{T} \mathbf{s}_{0} .
$$

Given two screws $\$_{1}$ and $\$_{2}$, the inner product between $\$_{1}$ and $\$_{2}$ is given as

$$
\$_{1} \cdot \$_{2}=s_{1} s_{2}+\epsilon\left(s_{1} s_{02}+s_{01} s_{2}\right) .
$$

Hereby, the dual part in (5) is defined as the reciprocal product, labeled as $\circ$, between two screws:

$$
\$_{1} \circ \$_{2}=s_{1} s_{02}+s_{01} s_{2} .
$$

Considering a wrench represented by $\$_{1}=f(\mathbf{f}+\in \boldsymbol{\tau})$ and a twist denoted by $\$_{2}=w(\mathbf{w}+\epsilon \mathbf{v})$, their reciprocal product, $\$_{1} \circ \$_{2}$, indicates the power generated by the wrench on the body that moves with the foregoing twist:

$$
\$_{1} \circ \$_{2}=f w(\mathbf{f} \cdot \mathbf{v}+\boldsymbol{\tau} \cdot \mathbf{w}),
$$

where $f$ and $w$ are the amplitudes of the wrench screw and twist screw, respectively.

The reciprocal product is a value indicating the instantaneous power between the wrench and twist. Particularly, if the value equals zero, $\$_{1} \circ \$_{2}=0$, it means that the relevant wrench applies no work on the rigid body in the direction of the twist. This is a singular solution in mathematics, corresponding to a singularity configuration in mechanism.

2.2. Approach to Singularity Identification. In a parallel robot, two important screw systems are involved, that is, the twist screw system and wrench screw system. The twists typically include permitted twist screws (PTSs, denoted by $\$_{P}$ ) and restricted twist screws (RTSs, denoted by $\$_{R}$ ). Meanwhile, the wrenches (forces and moments) are composed of transmission wrench screws (TWSs) and constraint wrench screws (CWSs) in a lower-mobility parallel robot. The TWS, denoted by $\$_{T}$, plays the role of transmitting the PTS, and the CWS, denoted by $\$_{C}$, is supposed to constrain the RTS in a mechanism. Figure 1 shows the interrelationship among the four subscrew systems, spanned by PTSs, RTSs, TWSs, and CWSs, respectively. The details about the calculation process have been depicted in $[26,27]$.
As is well known, the essential functions of a lowermobility parallel robot contain two sides of a coin: transmitting the expected motion/force from its input members to the output member and constraining unwanted motion/force between inputs and output of the parallel robot. Recalling (5), one can see that if the wrench, $\$_{1}=\$_{T}$, indicates the transmission wrench exerted on the rigid body, while the twist, $\$_{2}=\$_{P}$, indicates the underlying permitted motion, their reciprocal product signifies their instantaneous power. If the product is equal to zero, it means that the TWS applies no work in the direction of the PTS at this configuration of the parallel robot. Thus, the TWS loses the ability of transmitting the PTS stemming from a transmission singularity. In a similar way, if we set the wrench screw as $\$_{1}=\$_{C}$, the constraint wrench existed in the rigid body, and the twist screw as, $\$_{2}=\$_{R}$, the restricted motion, their reciprocal product could be used to identify constraint singularity. If the product between the CWS and RTS is zero, it means the RTS is not constrained by the CWS. At this configuration, the parallel robot adds one DOF, resulting in a constraint singularity. Hereby, the singularity of a parallel robot can be identified as transmission singularity and constraint singularity.

In addition, a parallel robot, as previously mentioned, contains the inputs and output, performing input twist screws and output twist screws, respectively. Normally, the input members are the actuators, and the output member is the moving platform. Inspired from the classification idea proposed by Zlatanov et al. [17], we can divide the transmission singularity into input transmission singularity (ITS) and output transmission singularity (OTS). Similarly, we consider the input and output constraint singularities (ICS and OCS), correspondingly. The four indices of singularities can be defined as

$$
\begin{gathered}
\text { ITS }=\left|\$_{T} \circ \$_{P I}\right|, \\
\text { OTS }=\left|\$_{T} \circ \$_{P O}\right|, \\
\text { ICS }=\left|\$_{C} \circ \$_{R I}\right|, \\
\text { OCS }=\left|\$_{C} \circ \$_{R O}\right|,
\end{gathered}
$$

where $\$_{P I}$ is the selected input PTS in each limb, $\$_{P O}$ is the output twist of the robot related to the analyzed transmission wrench, $\$_{R I}$ is the RTS in the limb, and $\$_{R O}$ is the output twist related to the constraint wrench of the robot.

Here we demonstrate the calculation process of $\$_{P O}$ and $\$_{R O}$ of the output using the tricks of "locking" and "releasing" in screw theory. In an $n$-DOF $(0<n<6)$ nonredundant parallel robot, the wrench screw system comprises $p$ TWSs and $q$ CWSs, where $p=n$ and $p+q=6$ are always established. In order to calculate the underlying $\$_{P O i}$ related to the $i$ th TWS, we can lock all other $(p-1)$ TWSs, thereby yielding five constraint wrenches including $(p-1)$ TWSs and $q$ CWSs. Then using reciprocal product in screw theory, one can calculate the related $\$_{\mathrm{PO} i}$ as

$$
\begin{gathered}
\$_{P O i} \circ \$_{T j}=0 \quad j=1,2, \ldots, p-1, \\
\$_{P O i} \circ \$_{C k}=0 \quad k=1,2, \ldots, q .
\end{gathered}
$$




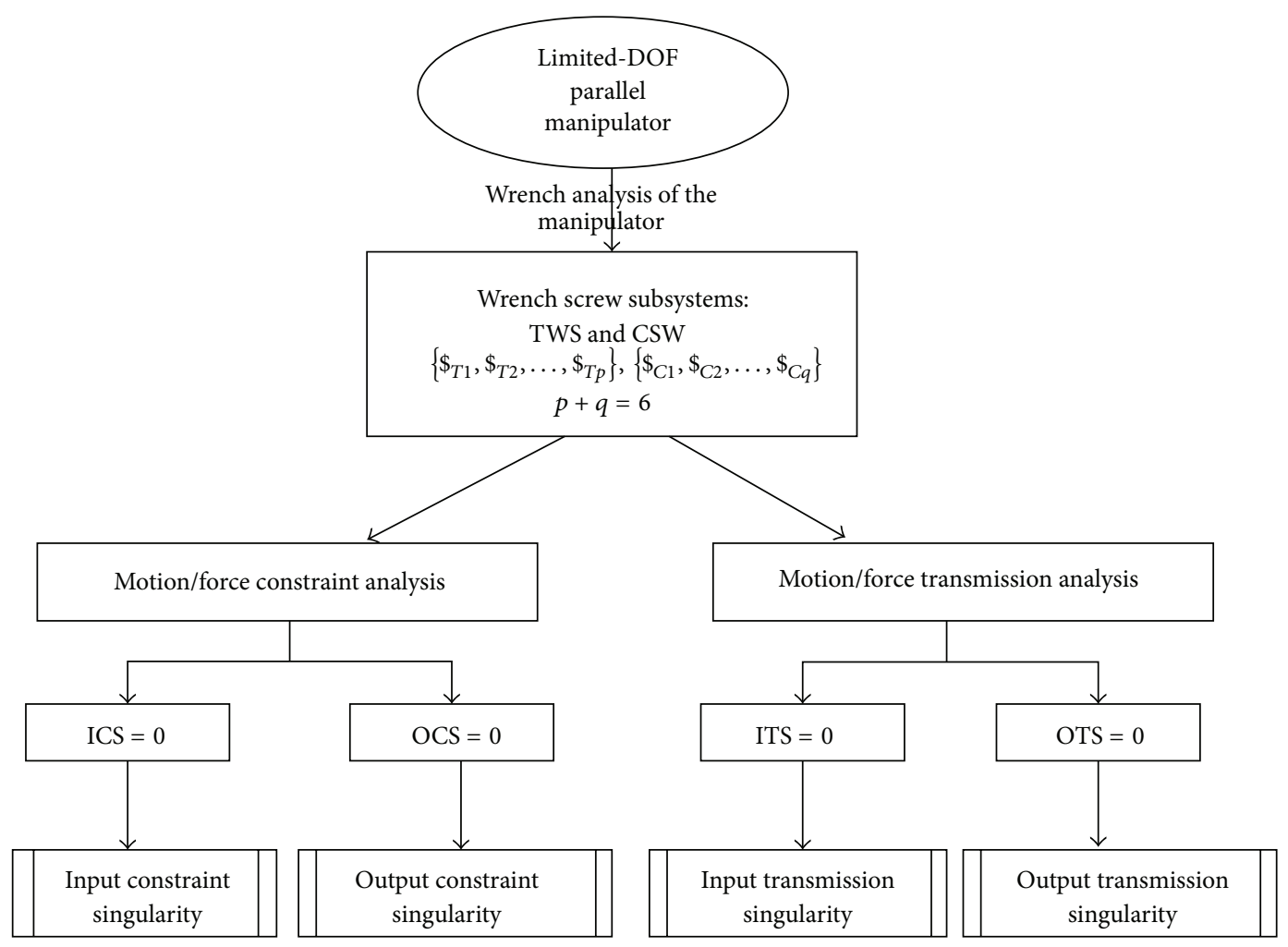

FIgURE 2: Process of singularity analysis in a lower-mobility parallel robot.

On the other hand, if we release the constraint of the $i$ th CWS while locking all the TWSs, the parallel robot is assumed to be a single-DOF one. The only imagined twist motion, $\$_{R O i}$, is considered to be the output RTS with regard to the $i$ th CWS. Hereby, we can achieve five constraint wrenches as well, that is, $p$ TWSs and $(q-1)$ CWSs. The related $\$_{R O i}$ could be calculated from

$$
\begin{gathered}
\$_{R O i} \circ \$_{T j}=0 \quad j=1,2, \ldots, p, \\
\$_{R O i} \circ \$_{C k}=0 \quad k=1,2, \ldots, q-1 .
\end{gathered}
$$

To sum up, the entire process of the singularity analysis of a lower-mobility parallel robot is depicted in Figure 2. Using (8)-(11), we can evaluate four classified types of singularities, that is, input constraint singularity, output constraint singularity, input transmission singularity, and output transmission singularity. Expressions (8)-(11) are nothing but the numerators of the existing performance indices proposed in $[18,19]$. The ranges of modified indices are zero to infinity. Clearly, the purpose here is to identify the singularity of a parallel robot; thus, only the indices being zero are meaningful. Four indices defined from the reciprocal product can be used not only to identify the singularities, but also to explain their physical meanings from the view of motion/force transmission and constraint performance.

\section{Examples}

We now turn towards illustrating how the classified singularities happen and function by analyzing some typical lower-mobility parallel robots. To realize this analysis, we should firstly study kinematics of the parallel mechanisms and describe their twists and wrenches via screw theory.

3.1. Input Constraint Singularity. Input constraint singularity is a new defined type of singularity, which was never involved in the literatures. It is the singularity caused by the constraint wrench in the limb of a parallel robot. At this singular configuration, the constraint force in input limbs cannot constrain unwanted motions as expected. We say that the constrainability of the limb becomes the worst resulting in an input constraint singularity. Hence, the parallel robot adds a DOF and loses control when its limbs lose the constrainability. Here, we take 3-US robot (Figure 3), a pure rotational mechanism, as an example to show details of the usage of ICS index.

Figure 3 is a 3-US parallel robot with three identical UStype limbs ( $\mathrm{U}$ denotes universal joint; $\mathrm{S}$ refers to spherical joint). Normally, $\mathrm{U}$ joint is a kinematic equivalent with two revolute joints with perpendicular axes, and $\mathrm{S}$ joint can be composed of three orthogonal revolute joints. Clearly, each US-type limb has five DOFs yielding one constraint force. The mechanism has three US-type limbs, achieving three constraint forces together. Thus, the mechanism performs three DOFs, that is, three rotational DOFs. In order to control it, three actuators should be settled for this mechanism. For the sake of simplicity, the revolute joints connected to the fixed base platform (in the U joint) are chosen to be actuated.

In order to calculate the ICS of the mechanism, we should firstly analyze the input US-type limbs via screw theory 


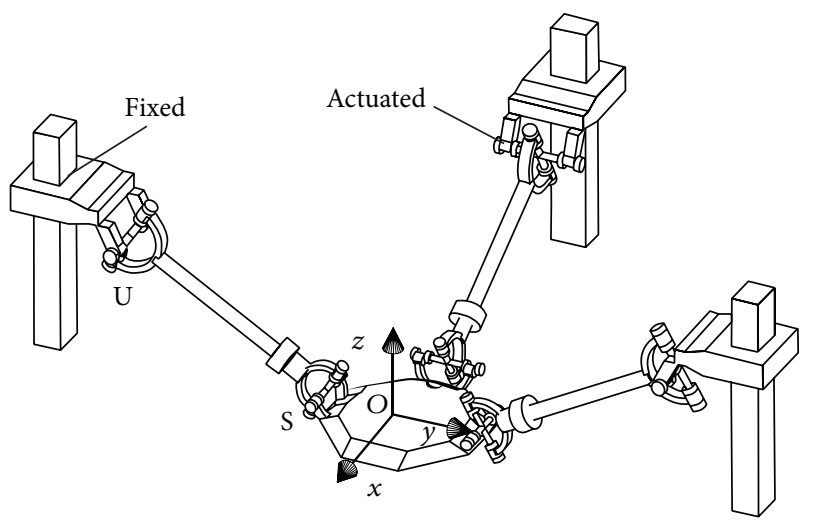

FIGURE 3: A 3-US parallel robot.

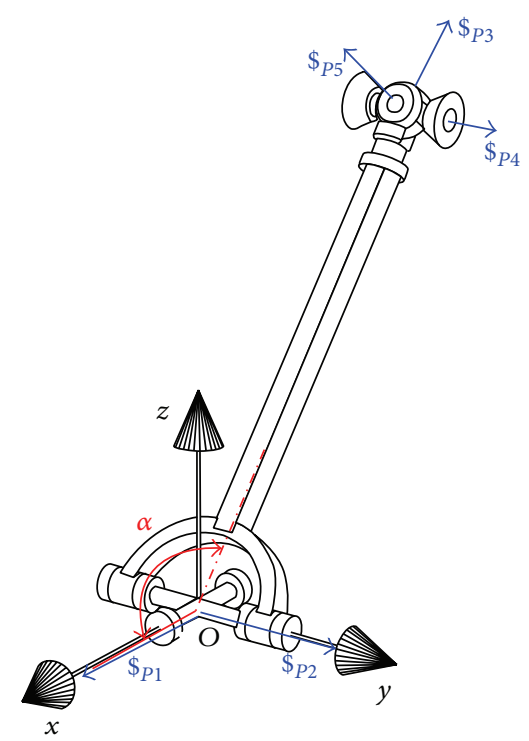

FIgure 4: A US-type limb.

(Figure 4). According to the relations depicted in Figure 1, it is intuitive to represent permitted twists, constraint wrenches, and restricted twists in this limb.

Figure 4 is a US-type limb, where a local coordinate system $O-x y z$ is established in the center point of $U$ joint. Under the coordinate, we can achieve five PTSs as

$$
\begin{gathered}
\$_{P 1}=(1,0,0)+\in(0,0,0) \\
\$_{P 2}=(0,1,0)+\epsilon(0,0,0) \\
\$_{P 3}=(\cos \alpha, 0, \sin \alpha)+\epsilon(0,0,0) \\
\$_{P 4}=(0,1,0)+\in(-l \sin \alpha, 0, l \cos \alpha) \\
\$_{P 5}=(-\sin \alpha, 0, \cos \alpha)+\in(0, l, 0) .
\end{gathered}
$$

Then, using reciprocal and dual relations among the twists and wrenches, we can achieve a CWS and an input RTS as

$$
\begin{gathered}
\$_{C}=(\cos \alpha, 0, \sin \alpha)+\epsilon(0,0,0), \\
\$_{R I}=(0,0,0)+\epsilon(0,0,1) .
\end{gathered}
$$

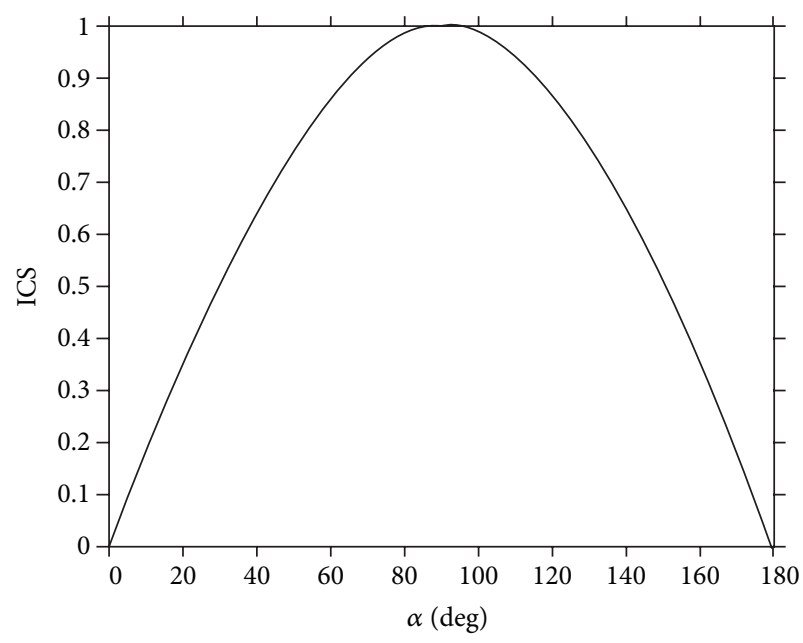

FIGURE 5: The relationship between the ICS and the angle, $\alpha$.

Recalling the defined ICS index (see (8)), we can calculate the ICS of the US-type limb as

$$
\mathrm{ICS}=\left|\$_{C} \circ \$_{R I}\right|=|\sin \alpha| .
$$

The relationship between ICS index and the angle, $\alpha$, is shown in Figure 5. From this figure, we can see that, when the angle equals 0 or $\pi$, the ICS equals zero indicting that the input constraint singularity happens at this configuration.

One thing should be noted: the singularity is only related to a whole parallel robot, rather than a serial limb. If we consider that a robot suffers from a singularity, the instantaneous DOF of the mechanism varies, rather than the motilities of the limb. Hence, we illustrate the singular configuration of 3-US parallel robot in Figure 6 caused by the zero index, ICS $=0$. Figure 6 shows an input constraint singularity configuration of 3-US parallel robot. At this configuration, the robot cannot constrain the translational motion along the direction of the $z$-axis, thereby adding a translational DOF along the $z$-axis. In other words, the robot cannot resist any external force along the direction of the $z$-axis, suffering from the poorest stiffness.

3.2. Output Constraint Singularity. Figure 7 shows a typical 3RPS parallel robot [28]. This robot has been analyzed by some researchers including its singularity property; that is, Briot and Bonev [29] discussed its singularity loci by calculating the Jacobian matrix. However, one type of singularity was missed in [29] that turns out the so-called output constraint singularity in this paper. Thereby we take 3-RPS parallel robot as an example to illustrate the usage of the introduced index OCS.

The mechanism has a moving platform that connects the base platform by three identical RPS limbs. Each RPS limb contains a revolute joint, prismatic joint, and spherical joint in turn. Three $\mathrm{P}$ joints are actuated. Considering that the robot has zero-torsion performance, we apply the tilt-andtorsion (T\&T) angles $(\varphi, \theta, \sigma)$ to describe the orientation of the moving platform, where $\varphi, \theta$, and $\sigma$ are referred to as 


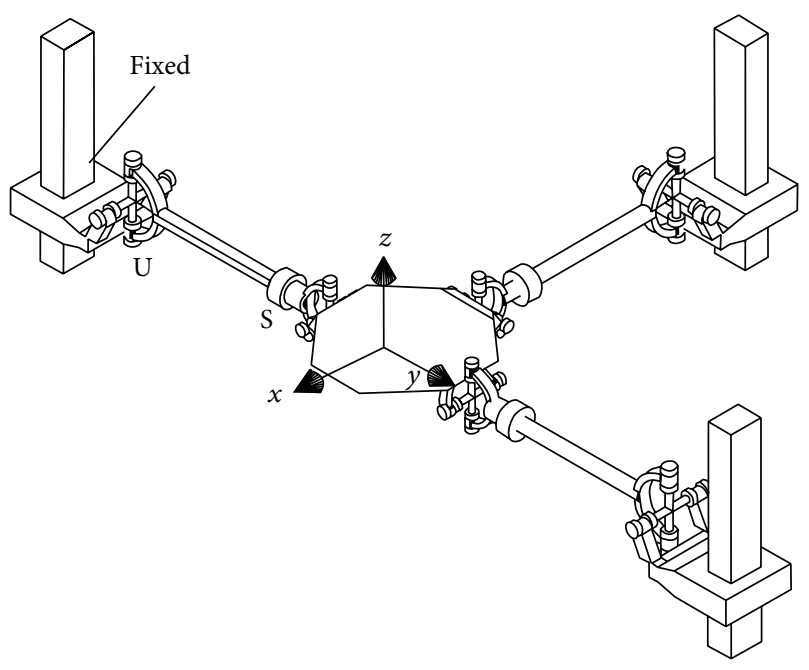

Figure 6: A singular configuration of the 3-US parallel robot.

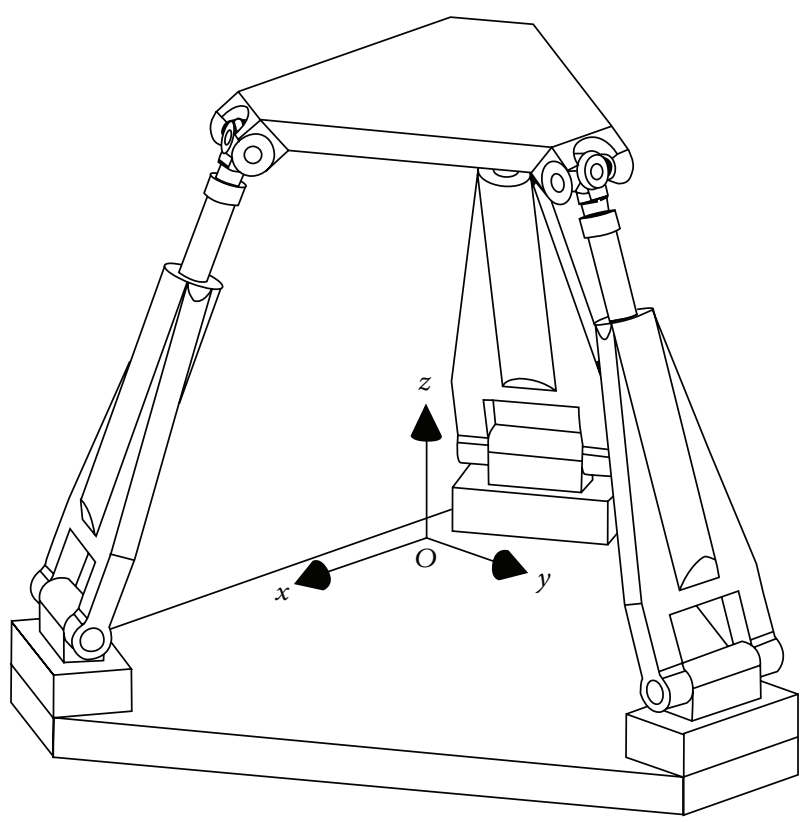

FIGURE 7: A 3-RPS parallel robot.

azimuth, tilt, and torsion angles, respectively [30]. Here, we let $\sigma$ be equal to 0 indicating zero torsion angle.

To evaluate output constraint performance of the mechanism, we should firstly analyze its twists and wrenches. Figure 8 shows one of the three identical RPS limbs. Screw theory is used to analyze the limb, wherein the $S$ joint can be regarded as a combination of three $\mathrm{R}$ joints with orthogonal axes. In the local coordinate frame attached to $\mathrm{R}$ joints in Figure 8, five PTSs can be easily achieved as

$$
\begin{gathered}
\$_{P 1}=(1,0,0)+\in(0,0,0), \\
\$_{P 2}=(0,1,0)+\epsilon(0, \cos \beta, \sin \beta), \\
\$_{P 3}=(1,0,0)+\epsilon(0, l \sin \beta, l \cos \beta), \\
\$_{P 4}=(0,1,0)+\epsilon(-l \sin \beta, 0,0), \\
\$_{P 5}=(0,0,1)+\epsilon(l \cos \beta, 0,0),
\end{gathered}
$$

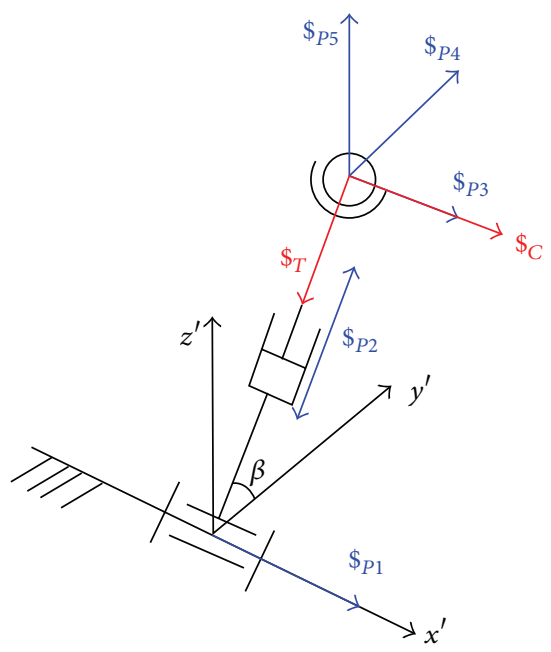

FIGURE 8: A RPS limb in 3-RPS mechanism.

where $\beta$ is the angle between the leg and $y^{\prime}$-axis and $l$ is the length of telescopic limb.

Then the CWS and TWS of the limb can be correspondingly calculated by reciprocal and dual properties as

$$
\begin{gathered}
\$_{C}=(1,0,0)+\epsilon(0, l \sin \beta,-l \cos \beta), \\
\$_{T}=(0, l \cos \beta, l \sin \beta)+\epsilon(0,0,0) .
\end{gathered}
$$

We can find that the CWS $\left(\$_{C}\right)$ indicates a pure force in the direction of the $x^{\prime}$-axis passing through the center of $S$ joint, and the TWS $\left(\$_{T}\right)$ is a pure force in the direction of the leg.

From the analysis of the RPS limb, we can conclude that 3-RPS robot contains three CWSs and three TWSs in sum. Then, according to (13) in Section 2, we can achieve the $i$ th RTS, $\$_{R O i}$, by releasing the $i$ th CWS at one time. Then three CWSs and their relevant RTSs can be calculated at hand. Recalling (11), we can identify the output constraint singularity by using OCS index.

Figure 9 shows the relationship between OCS and azimuth angle $\varphi$ by fixing the tilt angle $\theta=0$, and Figure 10 presents the relationship between OCS and tilt angle $\theta$ by fixing the azimuth angle $\varphi=0$. We can find that the CTP value keeps constant with the change of angle $\varphi$, while differing with the change of angle $\theta$. When the tilt angle $\theta=180^{\circ}$, CTP equals zero indicating the constraint singularity. That is to say, a 3-RPS parallel robot suffers from an output constraint singularity when its moving platform is upside down (Figure 11). We now turn towards explaining the physical meaning of this type of singularity from the view of motion/force constrainability. At this configuration, three CWSs (Figure 11) intersect at one point ( $C$ point); the robot cannot counterbalance any external torque on the intersection point behaving the poorest stiffness around the rotational axis. Thus, the mechanism adds one rotational DOF resulting in an output constraint singularity. 


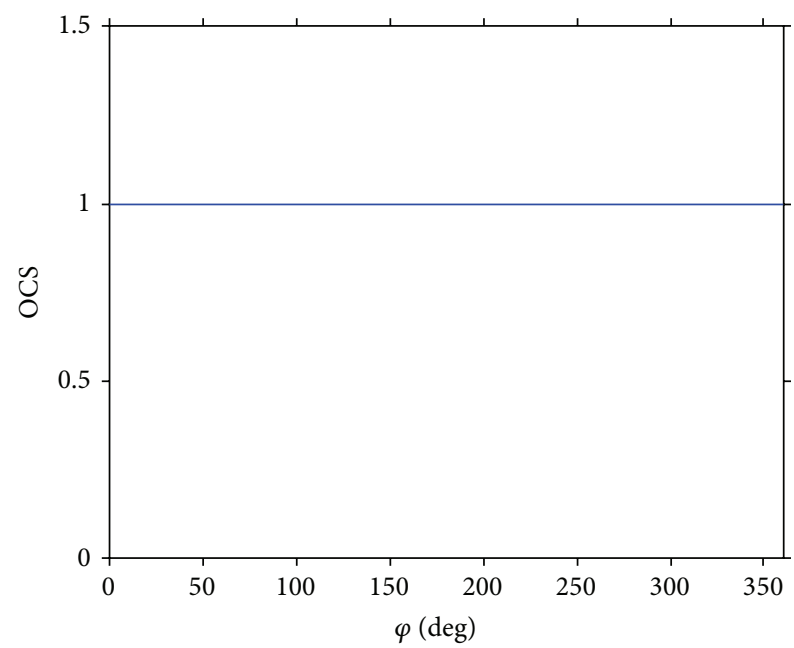

FIGURE 9: The relationship between the value of OCS and azimuth angle $\varphi$ by fixing tilt angle $\theta=0$.

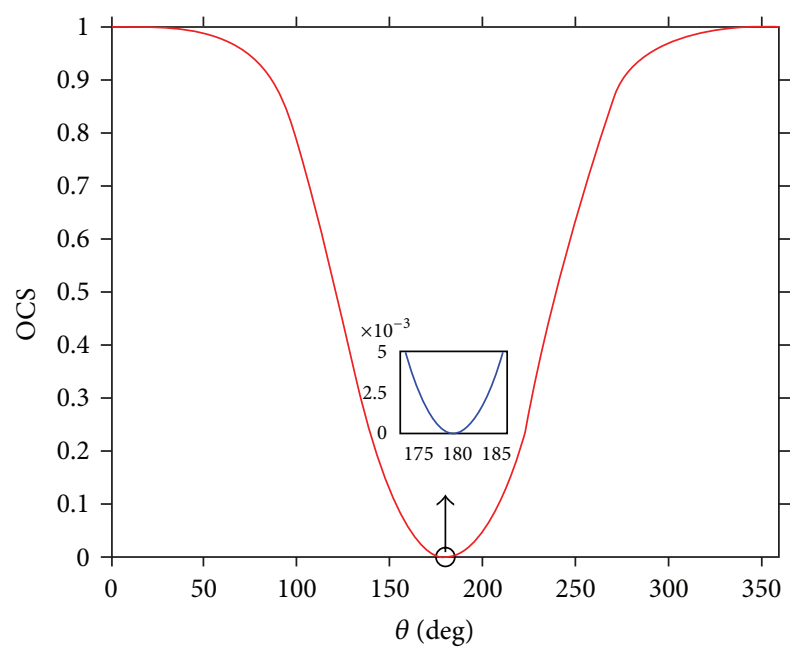

FIGURE 10: The relationship between the value of OCS and tilt angle $\theta$ by fixing azimuth angle $\varphi=0$.

3.3. Input Transmission Singularity. Input transmission singularity indicates that the motion/force cannot be transmitted out from inputs. This concept has been proposed in our previous research [25], wherein the index is used to analyze the closeness between a pose and the singularity. However, the aim of this work is to identify all possible singularities in a parallel robot; thus we modified the OCS index as (9).

Figure 12 is the kinematic schematic of a planar 3-RRR parallel robot. The mobile platform is connected to the base by three identical RRR limbs, each with three revolute joints and two bars. Figure 13 shows a planar RRR limb where the joint connected to the base is actuated. With respect to the global coordinate system $O-x y$, three PTSs can be expressed as

$$
\begin{aligned}
& \$_{P 1}=(0,0,1)+\epsilon\left(b_{1},-a_{1}, 0\right), \\
& \$_{P 2}=(0,0,1)+\epsilon\left(b_{2},-a_{2}, 0\right), \\
& \$_{P 3}=(0,0,1)+\epsilon\left(b_{3},-a_{3}, 0\right),
\end{aligned}
$$

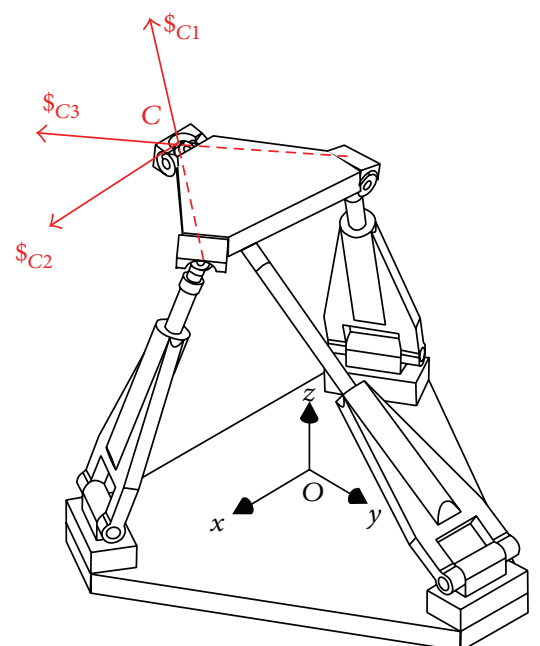

FIgURE 11: Output constraint singular configuration of 3-RPS robot.

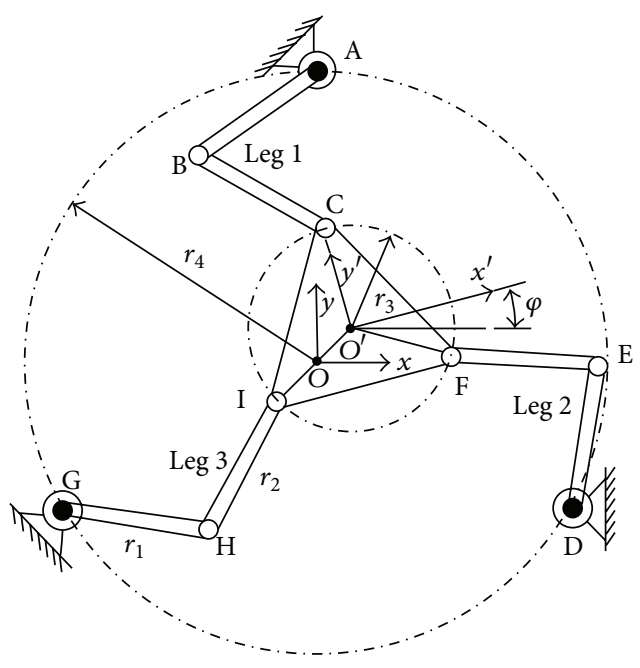

Actuator

Passive joint

FIGURE 12: A planar 3-RRR parallel robot.

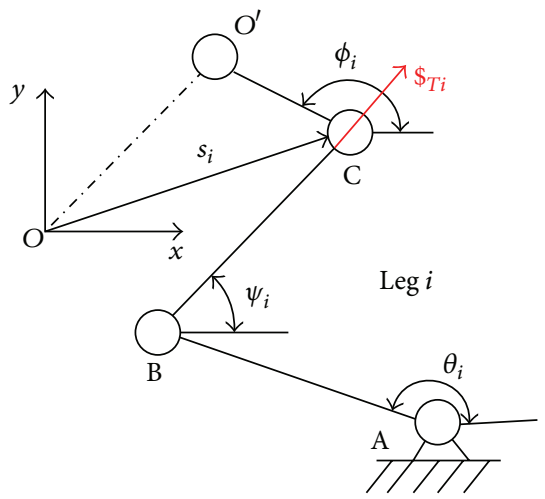

FIGURE 13: One RRR leg of the parallel robot. 


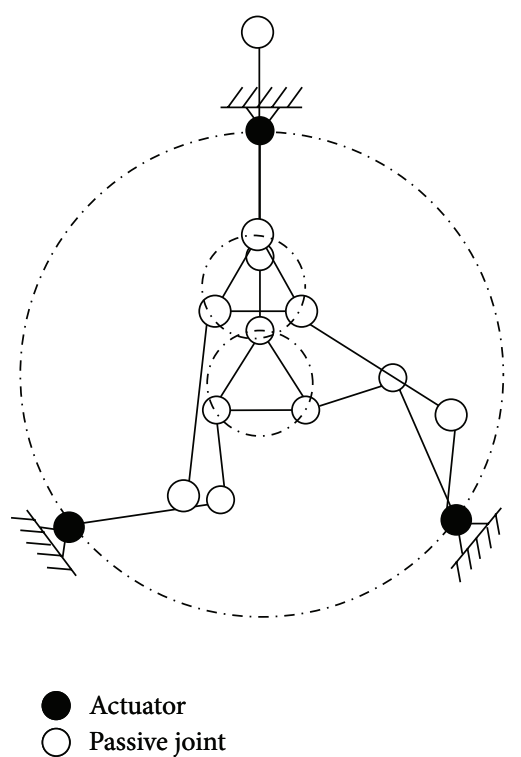

(a)

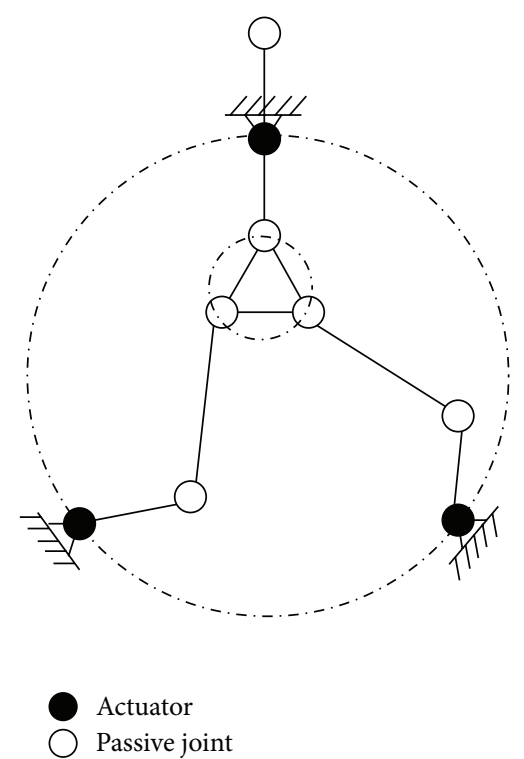

(b)

FIGURE 14: Input transmission singularity: (a) when $\theta_{1}=-90^{\circ}$ and $\psi_{1}=-90^{\circ}$; (b) when $\theta_{1}=90^{\circ}$ and $\psi_{1}=270^{\circ}$.

where $a_{m}, b_{m}, m=1,2,3$, are constants related to the instantaneous position of every kinematic pair.

Since the first joint connected to the base is actuated, the corresponding twist screw is an input PTS, which can be expressed as

$$
\$_{I}=\$_{P 1}=(0,0,1)+\in\left(b_{1},-a_{1}, 0\right) .
$$

Clearly, this planar RRR limb contains three twists, yielding no CWS in the plane. Hereby, we conclude that the 3-RRR parallel robot does not suffer from any constraint singularity. Each limb has its own TWS, transmitting the motion/force between input members and the output platform, which can be calculated as

$$
\$_{T i}=f_{i}+\epsilon\left(s_{i} \times f_{i}\right),
$$

where $f_{i}$ is a pure force vector moving along the direction of the centers of the two passive joints and $s_{i}$ represents a position vector at the original point of the proposed coordinate system (Figure 13).

According to (8), we can calculate the ITS index to elucidate the input transmission singularity:

$$
\text { ITS }=\$_{I} \circ \$_{T i}=\left|r_{1} \sin (\psi-\theta)\right|,
$$

where $r_{1}$ denotes the length of the leg $\mathrm{AB}, \psi$ is the angle of leg $\mathrm{BC}$ and the $x$-axis, and $\theta$ is the angle of leg $\mathrm{AB}$ and the $x$-axis (Figure 13).

From (20), one can find that ITS equals zero if and only if $\psi=\theta+i \pi(i=0,1,2, \ldots)$. That is to say, this singularity occurs when the active leg $\mathrm{AB}$ and passive leg $\mathrm{BC}$ are aligned. Indeed, one of the three legs is fully stretched-out or folded-back at the input singular configurations (Figures 14(a) and 14(b)). An infinitesimal rotation on the actuator of the link results

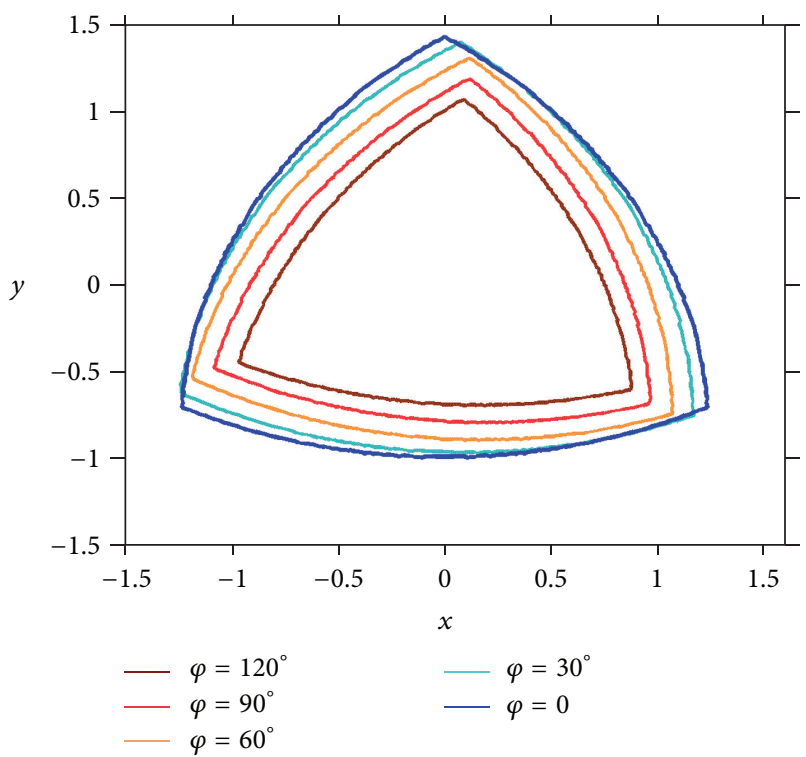

FIGURE 15: Input transmission singularity loci of a 3-RRR robot with rotational angle $\varphi$ equal to $0^{\circ}, 30^{\circ}, 60^{\circ}, 90^{\circ}$, and $120^{\circ}$.

in no output motion in the mobile platform. That means the motion cannot be transmitted from the inputs to the output member at such input transmission singular configurations. The mobile platform degenerates the translational DOF along direction of the limb.

Singularity loci comprise a series of continuous singularity points. Accordingly, input transmission singularity loci are made up of all points with ITS $=0$. The workspace enclosed by the singularity loci is always called the usable workspace. Figure 15 presents input transmission singularity loci of 


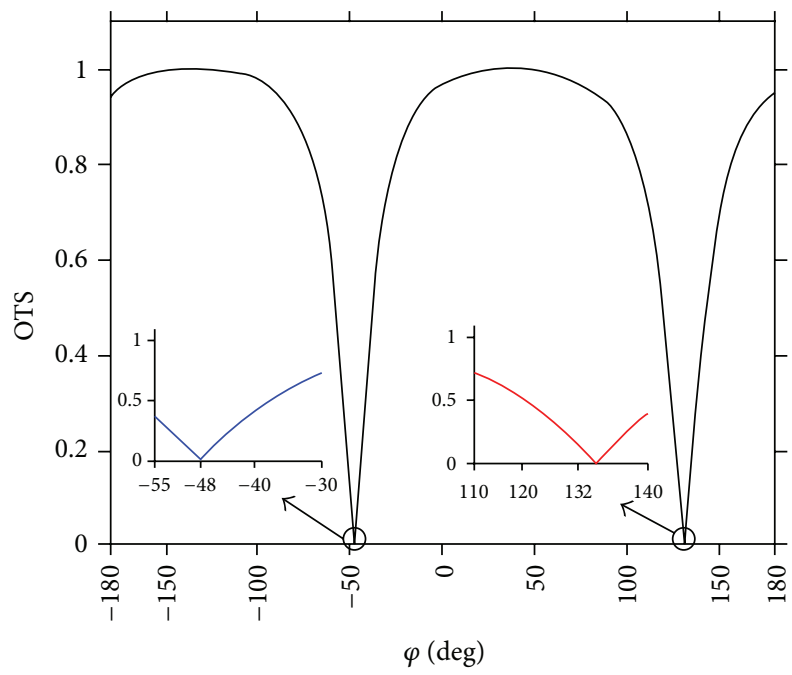

FIGURE 16: Relationship between OTS index and rotational angle $\varphi$ when the mobile platform is located at the original point.

3-RRR planar robot with the rotational angles $\varphi$ equal to $0^{\circ}$, $30^{\circ}, 60^{\circ}, 90^{\circ}$, and $120^{\circ}$, respectively. The usable workspace varies with the change of the rotational angle.

3.4. Output Transmission Singularity. The key to analyzing the output transmission singularity of a parallel robot is calculating the output PTS in relation to each TWS. We also take the planar 3-RRR parallel robot as an example to illustrate the usage of the defined index, OTS.

In a 3-RRR robot, as mentioned, there exist three TWSs in sum. We isolate the permitted output twist by locking any two TWSs while leaving one analyzed TWS free. At this moment, the 3-RRR robot could be regarded as a single-DOF mechanism. It is intuitive to see that the output PTS is only contributed by the analyzed TWS, $\$_{T i}$, whereas the other two locked TWSs apply no work in the direction of the twist. Then, from (12), we can derive this twist, $\$_{P O i}$ :

$$
\$_{T j} \circ \$_{P O i}=0 \quad(i, j=1,2,3, j \neq i) .
$$

The singularity depends not only on the pose of the mobile platform, but also on the position. There are uncountable configurable points in the usable workspace. For the sake of brevity, we here choose original point $(x, y)=(0,0)$ to evaluate the output transmission singularity. From (9), we can identify this type of singularity using the index, OTS:

$$
\mathrm{OTS}=\left|\$_{T i} \circ \$_{P O i}\right| \text {. }
$$

When the mobile platform of 3-RRR parallel robot is located at the original point, $(x, y)=(0,0)$, the OTS index varies with the rotational angle $\varphi$ of mobile platform. Figure 16 illustrates the relation between OTS index and the rotational angle $\varphi$ varying in $\left(-180^{\circ}, 180^{\circ}\right]$. From Figure 16, we can see that the OTS index equals zero when $\varphi_{1}=-48^{\circ}$ and $\varphi_{2}=132^{\circ}$, with regard to two output transmission singular configurations, respectively. Figures 17(a) and 17(b) show the output transmission singular configurations.

We now turn towards explaining the physical meaning in view of motion/force transmissibility. At the output transmission singularities, three TWSs converge to one point, resulting in the fact that any external torque along the axis through the convergence point cannot be counterbalanced by the TWSs. That is to say, the mechanism behaves the poorest stiffness along the direction of the normal axis. At such configurations, the mobile platform adds a rotational DOF when all the actuators are locked.

It should be noted that there exist some configurations that happen to suffer from more than one type of singularity. That is to say, at some configurations, both input and output transmission or constraint performances are poor, yielding different kinds of singularities at the same time. We can demonstrate that from the 3-RRR case with other parameters; that is, two legs in each chain are equal, $r_{1}=r_{2}$, and the radii of the base and mobile platforms are equal to each other, $r_{3}=r_{4}$. In such architecture, the base platform has the same size as the mobile platform. Using the proposed approach presented in Section 2, we can calculate the ITS and OTS of this manipulator. Figure 18 shows the relationships among the two indices and the rotational angle of the mobile platform, in which the ITS and OTS values are both equal to zero when the rotational angle $\varphi$ equals zero. In other words, the 3-RRR parallel manipulator suffers from both input and output transmission singularities (Figure 19) at one configuration, in which the mobile and base platforms coincide with each other. At this transmission singular configuration, the transmission wrenches cannot transmit any motion or force between the input members and the output member. Thus, the actuators could no longer drive the mobile platform.

\section{Conclusion}

In this work, we propose a new approach to identifying the singularities of lower-mobility parallel robots, which is an important issue in engineering. Although certain existing approaches have been introduced to analyze singularities from different aspects, this work provides an alternative viewpoint and approach to singularity identification and classification. The theory of screws is used as the mathematical foundation to identify singularities considering the motion/force transmissibility and constrainability. Meanwhile, we classify the singularities into four types based on the transmission and constraint performance in both the input and output members. Using screw theory, four generalized indices, ICS, OCS, ITS, and OTS, are proposed to identify input constraint singularity, output constraint singularity, input transmission singularity, and output transmission singularity, respectively. The approach stands out with some merits including that it can identify all possible singularities, as well as explaining their physical meanings from the view of essential properties of parallel robots. Furthermore, based on the proposed approach and indices, we analyze the singularities of 3-US, 


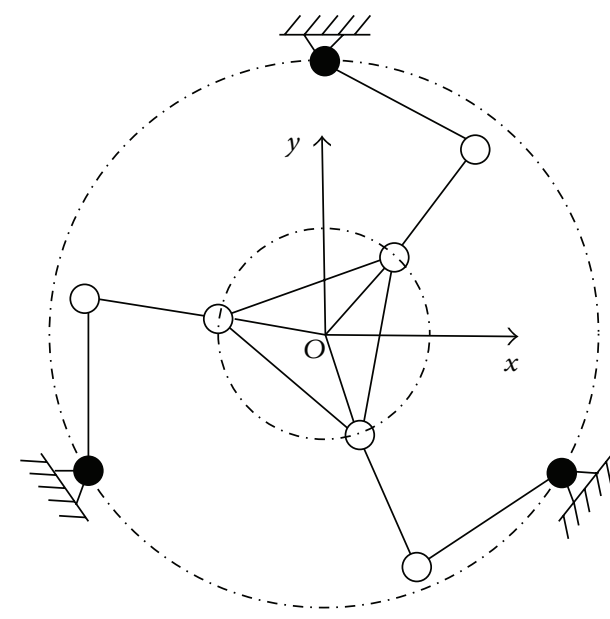

Actuator

Passive joint

(a)

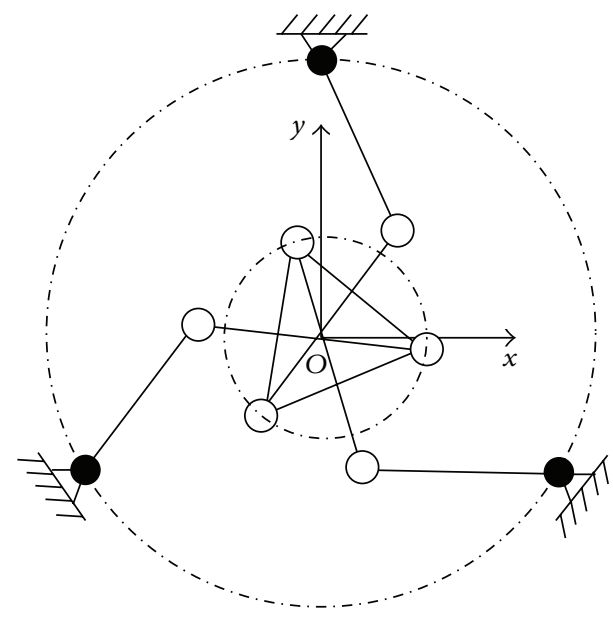

Actuator

Passive joint

FIGURE 17: Output transmission singularity configurations with rotational angles (a) $\varphi_{1}=-48^{\circ}$ and (b) $\varphi_{2}=132^{\circ}$.

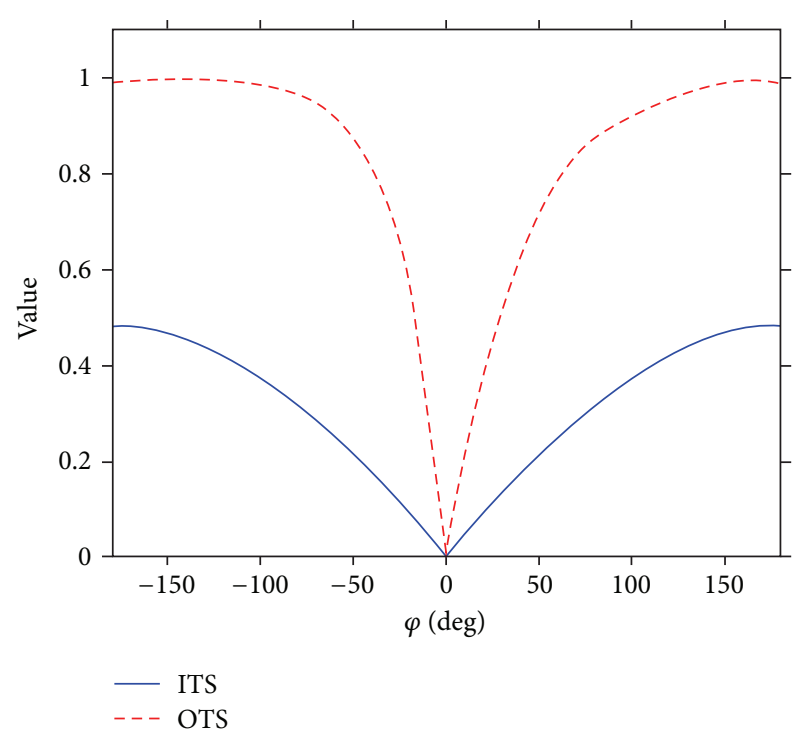

FIGURE 18: Relationships among ITS and OTS values and the rotational angle $\varphi$.

3-RPS, and 3-RRR parallel robots. All the singularity results are meaningful to facilitate better understanding and usage of the mechanisms in engineering. In addition, the elimination of these four classified types of singularities will be investigated in our further research.

\section{Conflict of Interests}

The authors declare that there is no conflict of interests regarding the publication of this paper.

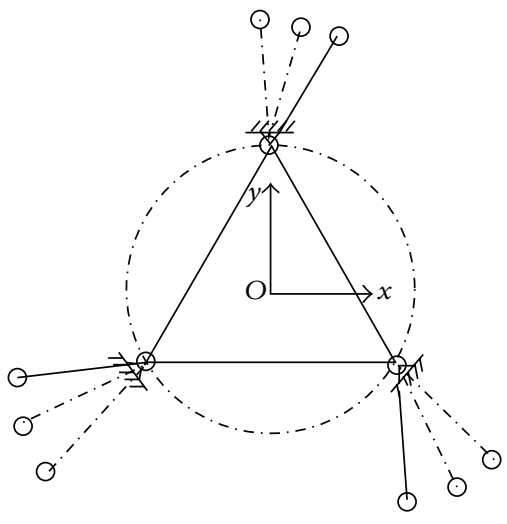

Actuator

Passive joint

FIGURE 19: Input and output transmission singular configuration of the 3-RRR manipulator with parameters $r_{1}=r_{2}$ and $r_{3}=r_{4}$.

\section{Acknowledgment}

This work was supported by the National Natural Science Foundation of China (Grant nos. 51375251 and 51135008).

\section{References}

[1] Y. Patel and P. George, "Parallel manipulators applications-a survey," Modern Mechanical Engineering, vol. 2, no. 3, pp. 5764, 2012.

[2] C. Gosselin and J. Angeles, "Singularity analysis of closedloop kinematic chains," IEEE Transactions on Robotics and Automation, vol. 6, no. 3, pp. 281-290, 1990. 
[3] Q. Xu and Y. Li, "Design and analysis of a new singularityfree three-prismatic-revolute-cylindrical translational parallel manipulator," Proceedings of the Institution of Mechanical Engineers, Part C: Journal of Mechanical Engineering Science, vol. 221, no. 5, pp. 565-577, 2007.

[4] N. Shvalb, M. Shoham, H. Bamberger, and D. Blanc, "Topological and kinematic singularities for a class of parallel mechanisms," Mathematical Problems in Engineering, vol. 2009, Article ID 249349, 12 pages, 2009.

[5] F. C. Park and J. W. Kim, "Singularity analysis of closed kinematic chains," Journal of Mechanical Design, Transactions of the ASME, vol. 121, no. 1, pp. 32-38, 1999.

[6] K. H. Hunt, Kinematic Geometry of Mechanisms, Oxford University Press, London, UK, 1978.

[7] F. Hao and J. M. McCarthy, "Conditions for line-based singularities in spatial platform manipulators," Journal of Robotic Systems, vol. 15, no. 1, pp. 43-55, 1998.

[8] J.-P. Merlet, "Singular configurations of parallel manipulators and Grassmann geometry," International Journal of Robotics Research, vol. 8, no. 5, pp. 45-56, 1989.

[9] P. Ben-Horin and M. Shoham, "Singularity condition of six-degree-of-freedom three-legged parallel robots based on Grassmann-Cayley Algebra," IEEE Transactions on Robotics, vol. 22, no. 4, pp. 577-590, 2006.

[10] J. K. Davidson and K. H. Hunt, Robots and Screw Theory: Applications of Kinematics and Statics to Robotics, Oxford University Press, London, UK, 2004.

[11] O. Ma and J. Angeles, "Architecture singularities of platform manipulators," in Proceedings of the IEEE International Conference on Robotics and Automation, pp. 1542-1547, Sacramento, Calif, USA, April 1991.

[12] I. A. Bonev, D. Zlatanov, and C. M. Gosselin, "Singularity analysis of 3-DOF planar parallel mechanisms via screw theory," Journal of Mechanical Design, Transactions of the ASME, vol. 125, no. 3, pp. 573-581, 2003.

[13] L. W. Tsai, Robot Analysis: The Mechanics of Serial and Parallel Manipulators, Wiley-Interscience, New York, NY, USA, 1999.

[14] G. Gogu, "Families of $6 \mathrm{R}$ orthogonal robotic manipulators with only isolated and pseudo-isolated singularities," Mechanism and Machine Theory, vol. 37, no. 11, pp. 1347-1375, 2002.

[15] G. Liu, Y. Lou, and Z. Li, "Singularities of parallel manipulators: a geometric treatment," IEEE Transactions on Robotics and Automation, vol. 19, no. 4, pp. 579-594, 2003.

[16] Y. Fang and L.-W. Tsai, "Structure synthesis of a class of 4-DoF and 5-DoF parallel manipulators with identical limb structures," The International Journal of Robotics Research, vol. 21, no. 9, pp. 799-810, 2002.

[17] D. Zlatanov, R. G. Fenton, and B. Benhabib, "Identification and classification of the singular configurations of mechanisms," Mechanism and Machine Theory, vol. 33, no. 6, pp. 743-760, 1998.

[18] Y. Li and Q. Xu, "Kinematic analysis and design of a new 3DOF translational parallel manipulator," Journal of Mechanical Design, Transactions of the ASME, vol. 128, no. 4, pp. 729-737, 2006.

[19] J. Wang, C. Wu, and X.-J. Liu, "Performance evaluation of parallel manipulators: motion/force transmissibility and its index," Mechanism and Machine Theory, vol. 45, no. 10, pp. 1462-1476, 2010.

[20] X.-J. Liu, X. Chen, and M. Nahon, "Motion/force constrainability analysis of lower-mobility parallel manipulators," Journal of Mechanisms and Robotics, vol. 6, no. 3, Article ID 031006, 2014.
[21] Y. F. Fang and L. W. Tsai, "Enumeration of a class of overconstrained mechanisms using the theory of reciprocal screws," Mechanism and Machine Theory, vol. 39, no. 11, pp. 1175-1187, 2004.

[22] H. R. Fang, Y. F. Fang, and K. T. Zhang, "Reciprocal screw theory based singularity analysis of a novel 3-DOF parallel manipulator," Chinese Journal of Mechanical Engineering, vol. 25, no. 4, pp. 647-653, 2012.

[23] X. W. Kong and C. M. Gosselin, “ Type synthesis of 3-DOF translational parallel $\mathrm{m}$ anipulators based on screw theory," Journal of Mechanical Design, Transactions of the ASME, vol. 126, no. 1, pp. 83-92, 2004.

[24] Q. Xu and Y. Li, "An investigation on mobility and stiffness of a 3-DOF translational parallel manipulator via screw theory," Robotics and Computer-Integrated Manufacturing, vol. 24, no. 3, pp. 402-414, 2008.

[25] X.-J. Liu, C. Wu, and J. Wang, "A new approach for singularity analysis and closeness measurement to singularities of parallel manipulators," ASME Journal of Mechanical and Robotics, vol. 4, no. 4, Article ID 041001, 2012.

[26] G. R. Veldkamp, "On the use of dual numbers, vectors and matrices in instantaneous, spatial kinematics," Mechanism and Machine Theory, vol. 11, no. 2, pp. 141-156, 1976.

[27] T. Huang, H. T. Liu, and D. G. Chetwynd, "Generalized Jacobian analysis of lower mobility manipulators," Mechanism and Machine Theory, vol. 46, no. 6, pp. 831-844, 2011.

[28] C.-C. Kao and T.-S. Zhan, "Modified PSO method for robust control of 3RPS parallel manipulators," Mathematical Problems in Engineering, vol. 2010, Article ID 302430, 25 pages, 2010.

[29] S. Briot and I. A. Bonev, "Singularity analysis of zero-torsion parallel mechanisms," in Proceedings of the IEEE/RSJ International Conference on Intelligent Robots and Systems (IROS '08), pp. 1952-1957, Nice, France, September 2008.

[30] X.-J. Liu and I. A. Bonev, "Orientation capability, error analysis, and dimensional optimization of two articulated tool heads with parallel kinematics," Journal of Manufacturing Science and Engineering, vol. 130, no. 1, pp. 0110151-0110159, 2008. 


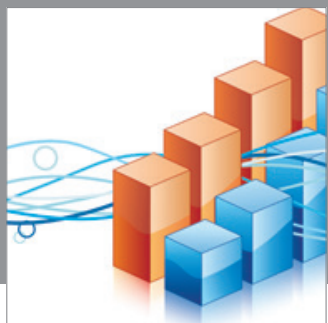

Advances in

Operations Research

mansans

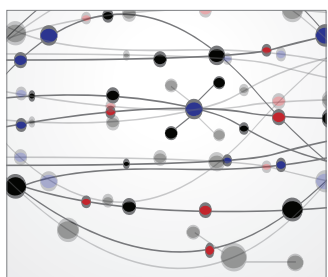

The Scientific World Journal
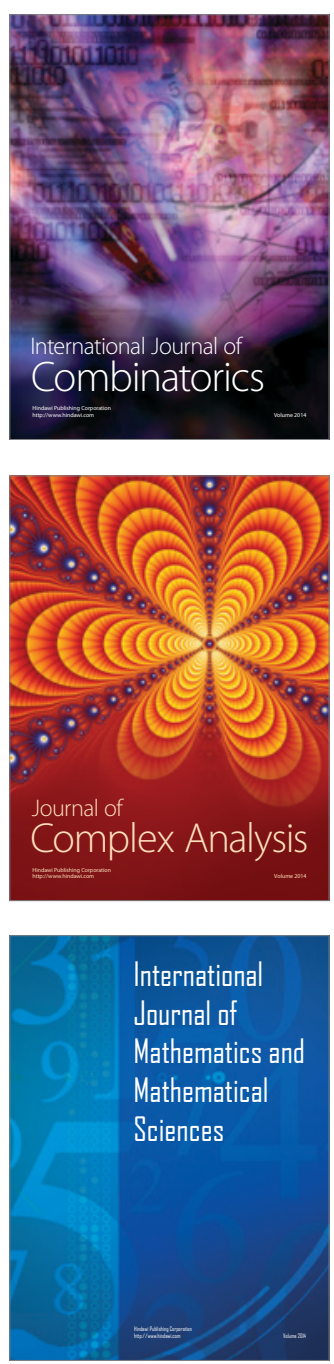
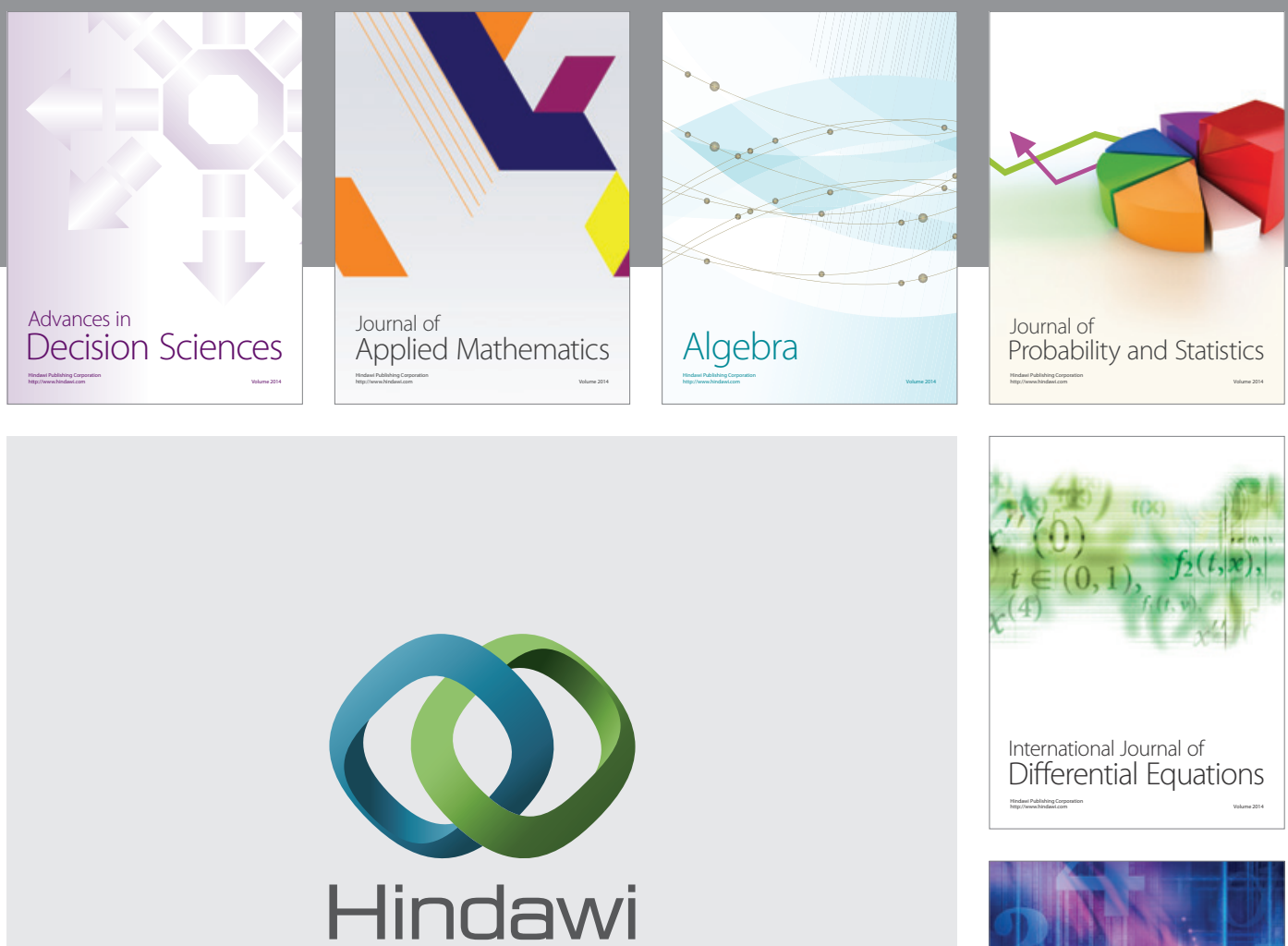

Submit your manuscripts at http://www.hindawi.com
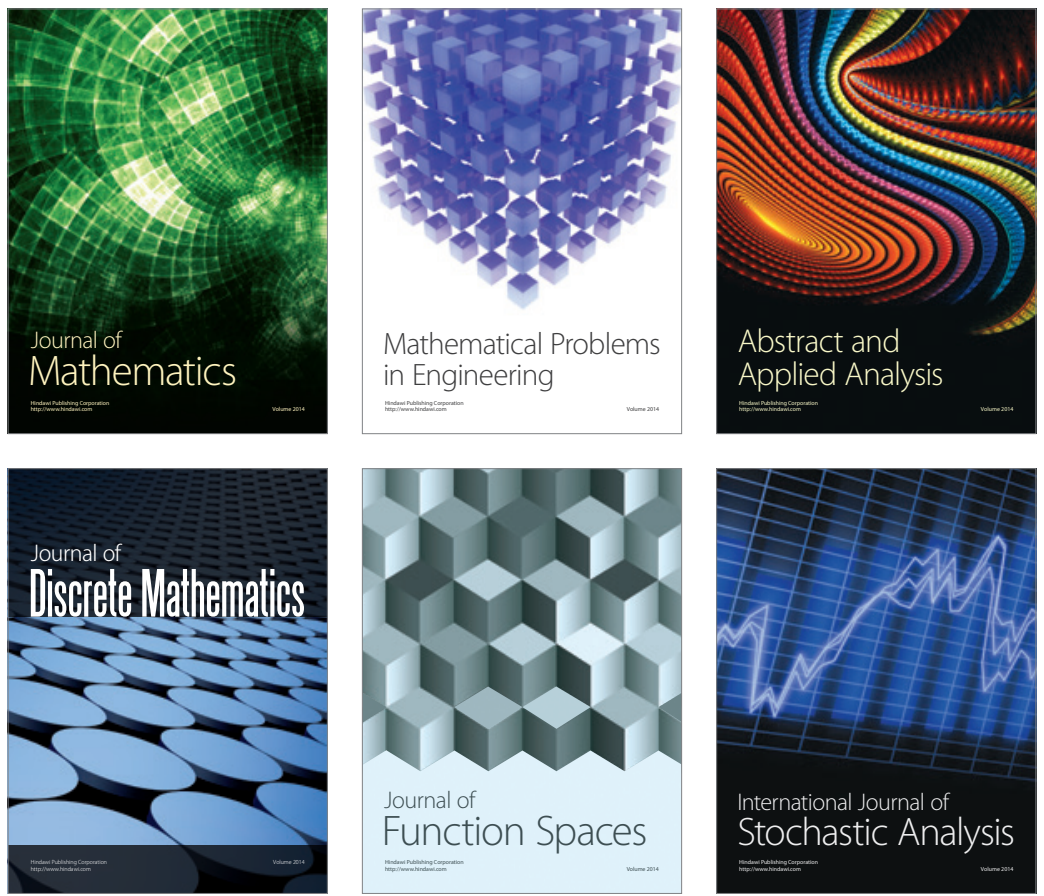

Journal of

Function Spaces

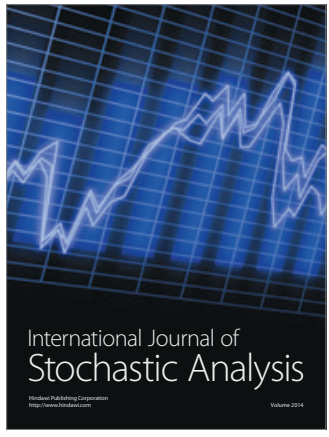

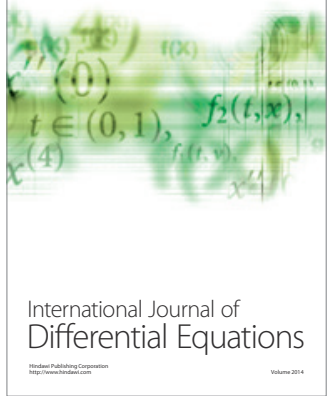
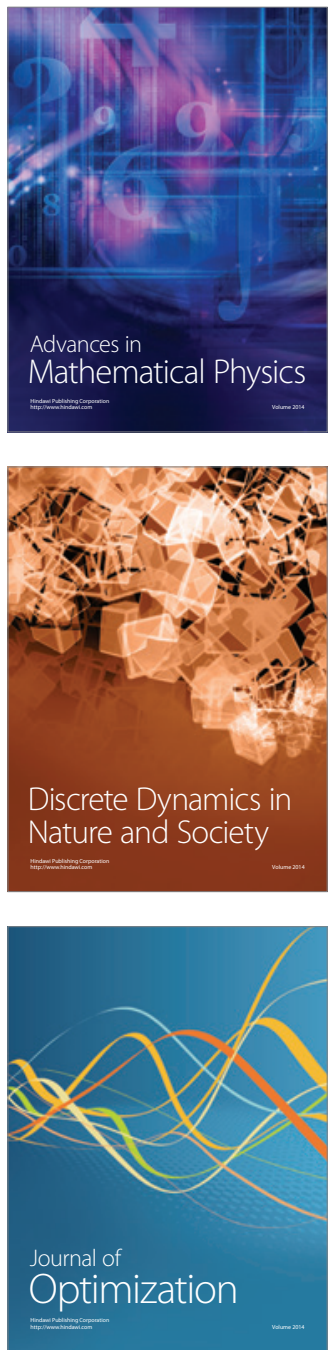